\title{
Regulation of osteogenic differentiation of human adipose-derived stem cells by controlling electromagnetic field conditions
}

\author{
Kyung Shin Kang ${ }^{1}$, Jung Min Hong ${ }^{1}$, Jo A Kang ${ }^{2}$, Jong-Won Rhie ${ }^{2}$, Young Hun Jeong ${ }^{3}$ and Dong-Woo Cho ${ }^{1,4}$ \\ Many studies have reported that an electromagnetic field can promote osteogenic differentiation of mesenchymal stem cells. \\ However, experimental results have differed depending on the experimental and environmental conditions. Optimization of \\ electromagnetic field conditions in a single, identified system can compensate for these differences. Here we demonstrated that \\ specific electromagnetic field conditions (that is, frequency and magnetic flux density) significantly regulate osteogenic \\ differentiation of adipose-derived stem cells (ASCs) in vitro. Before inducing osteogenic differentiation, we determined ASC \\ stemness and confirmed that the electromagnetic field was uniform at the solenoid coil center. Then, we selected positive \\ $(30 / 45 \mathrm{~Hz}, 1 \mathrm{mT})$ and negative $(7.5 \mathrm{~Hz}, 1 \mathrm{mT})$ osteogenic differentiation conditions by quantifying alkaline phosphate (ALP) \\ mRNA expression. Osteogenic marker (for example, runt-related transcription factor 2) expression was higher in the $30 / 45 \mathrm{~Hz}$ \\ condition and lower in the $7.5 \mathrm{~Hz}$ condition as compared with the nonstimulated group. Both positive and negative regulation \\ of ALP activity and mineralized nodule formation supported these responses. Our data indicate that the effects of the \\ electromagnetic fields on osteogenic differentiation differ depending on the electromagnetic field conditions. This study \\ provides a framework for future work on controlling stem cell differentiation.
}

Experimental \& Molecular Medicine (2013) 45, e6; doi:10.1038/emm.2013.11; published online 25 January 2013

Keywords: adipose-derived stem cells; electromagnetic field; frequency; magnetic flux density; optimization; osteogenic differentiation

\section{INTRODUCTION}

Low-frequency, low-energy, electromagnetic fields are commonly used to promote bone fracture healing. ${ }^{1,2}$ These electromagnetic fields enhance osteogenesis, decrease osteoporosis ${ }^{3-6}$ and regulate diverse osteoblastic responses for osteogenesis in vitro. DNA synthesis can also be enhanced by electromagnetic fields. ${ }^{7,8}$ In addition, electromagnetic fields increase osteogenic marker gene expression during differentiation ${ }^{9}$ and affect calcified matrix production during mineralization. ${ }^{10}$

Two parameters primarily characterize electromagnetic field properties: frequency and magnetic flux density. The magnetic flux density used in most previous studies to induce osteogenesis varied from 0.1 to $3 \mathrm{mT}^{6,8,9,11-13}$ Such studies have shown that a low-frequency electromagnetic field is more effective than a static field in causing biological effects. ${ }^{14,15}$ Frequencies used for osteogenesis varied from 7.5 to $75 \mathrm{~Hz}^{5-8,11,16,17}$ Unlike bio-mimetic stimuli (for example, cyclic strain and shear stress), this time-varying electromagnetic field does not have logical cues for each stimulation parameter. Thus, studies aimed at identifying optimal parameters, at least within the ranges of previous investigations, are required.

Previous studies on osteogenesis using electromagnetic fields have been performed under various conditions, ${ }^{1,2,4-10,12,13,15-17}$ and the results have differed depending on the experimental and environmental conditions. These differences may be attributable to different experimental systems. Moreover, authors have rarely discussed why they selected specific conditions for stimulating cells in their studies. Therefore, these conditions should be optimized

${ }^{1}$ Department of Mechanical Engineering, Pohang University of Science and Technology (POSTECH), Pohang, Korea; '2Department of Plastic Surgery, College of Medicine, The Catholic University of Korea, Seoul, Korea; ${ }^{3}$ Department of Mechanical Engineering, Korea Polytechnic University, Siheung, Korea and ${ }^{4}$ Division of Integrative Biosciences and Biotechnology, Pohang University of Science and Technology (POSTECH), Pohang, Korea

Correspondence: Professor YH Jeong, Department of Mechanical Engineering, Korea Polytechnic University, 2121 Jeongwang-dong, Siheung, Gyeonggi-do 429-793, Korea.

E-mail:yhjeong@kpu.ac.kr

or Professor D-W Cho, Department of Mechanical Engineering and Center for Rapid Prototyping Based 3D Tissue/Organ Printing, Pohang University of Science and Technology (POSTECH), San 31, Hyoja-dong, Nam-gu, Pohang 790-751, Korea.

E-mail:dwcho@postech.ac.kr

Received 29 August 2012; revised 29 October 2012; accepted 5 November 2012 
in a single, identified system where the experimental and environmental conditions are fixed.

We examined the effects of various electromagnetic field conditions on the osteogenic differentiation of mesenchymal stem cells. We used adipose-derived stem cells (ASCs) because ASCs easily attach to surfaces and proliferate rapidly. ${ }^{18}$ In addition, ASCs can be derived from fat tissue, which afforded us an abundant source of cells. ASCs also have osteogenic potential, among various differentiation lineages, ${ }^{18-22}$ and are known to play a role in bone regeneration, including the acceleration of bone fracture healing ${ }^{23,24}$ and promotion of bone regeneration in cases of calvarial defects without chemical induction. ${ }^{19}$

Here, we observed the effects of an electromagnetic field under various conditions on osteogenic differentiation of ASCs. A fixed electromagnetic field distribution was used to provide uniform field effects on cells. Among various combinations of frequency and magnetic flux density, we selected and investigated specific conditions that had positive (30$45 \mathrm{~Hz}, 1 \mathrm{mT})$ or negative $(7.5 \mathrm{~Hz}, 1 \mathrm{mT})$ effects on ASC differentiation.

\section{MATERIALS AND METHODS}

\section{Ethics statement}

This study was approved by the Institutional Review Board of Seoul St Mary's Hospital and conformed to the principles expressed in the Declaration of Helsinki. All patients provided written informed consent for the collection of cells and subsequent analysis.

\section{Isolation, culture and characterization of ASCs}

ASCs were isolated and cultured as previously described. ${ }^{25}$ The isolated ASCs were cultured in Dulbecco's modified Eagle's medium (Gibco BRL, Grand Island, NY, USA) containing 10\% (v/v) fetal bovine serum (Gibco BRL), $100 \mathrm{U} \mathrm{ml}^{-1}$ penicillin and $100 \mu \mathrm{g} \mathrm{ml}^{-1}$ streptomycin (Gibco BRL) at $37^{\circ} \mathrm{C}$ in a humidified atmosphere containing 5\% $\mathrm{CO}_{2}$. All experiments were performed at passage three. To induce osteogenic differentiation, the culture medium was changed to osteogenic induction medium (OM) containing $10 \mathrm{~nm}$ dexamethasone (Sigma-Aldrich, St Louis, MO, USA), $10 \mathrm{~mm}$ $\beta$-glycerol phosphate (Sigma-Aldrich) and $50 \mu \mathrm{g} \mathrm{ml}^{-1}$ ascorbic acid-2-phosphate (Sigma-Aldrich).

For flow cytometry, cells were harvested with trypsin/EDTA and washed in $1 \%$ bovine serum albumin (Pierce, Rockford, IL, USA). Cells were then incubated with fluorescently labeled monoclonal antibodies against CD13, CD29, CD34, CD45, CD90 and CD105 (Serotec, Oxford, UK) for $30 \mathrm{~min}$ at $4{ }^{\circ} \mathrm{C}$ and analyzed using a flow cytometer (Becton Dickinson, Mountain View, CA, USA).

\section{Exposure of ASCs to the electromagnetic field}

A solenoid coil was used because it could generate a uniform electromagnetic field. A custom-made solenoid coil had the wire wound 7300 times and the DC resistance was $450 \Omega$ for $10 \mathrm{mT}$. The inner diameter and length were 180 and $400 \mathrm{~mm}$, respectively. We used 3-axis magnetic field sensors (MFS-3A, Ametes, San Carlos, CA, USA) to measure the magnetic flux density inside the coil. The 24-well plates were used with a cell density of $2 \times 10^{4}$ cells per well. Cells were exposed to the electromagnetic field for $8 \mathrm{~h}$ per day in a humidified incubator at $37^{\circ} \mathrm{C}$ under a $5 \% \mathrm{CO}_{2}$ atmosphere. All experiments were conducted in the conditions without heat generation.
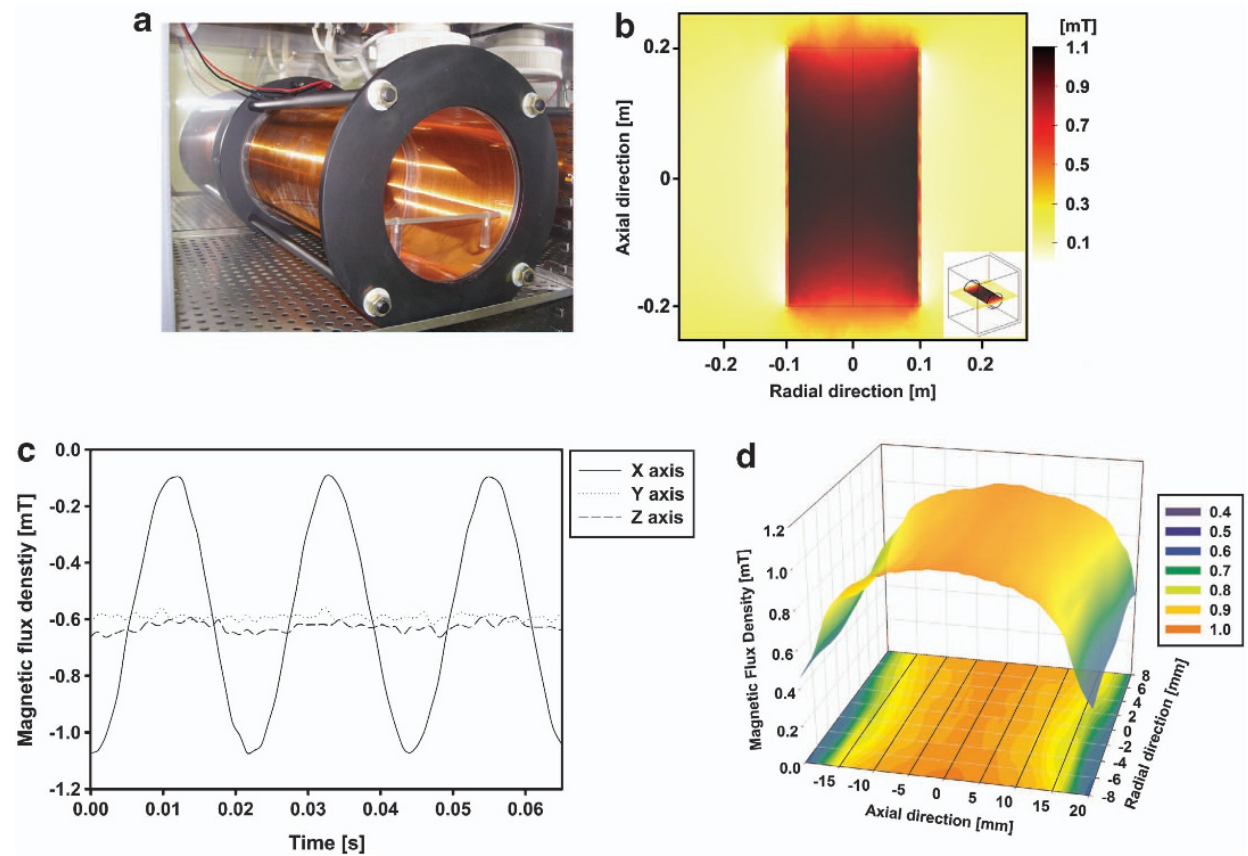

Figure 1 Identification of the electromagnetic field generated by a solenoid coil. (a) The solenoid coil, which generated the electromagnetic field, was located inside a $\mathrm{CO}_{2}$ incubator. The inner diameter and length were 180 and $400 \mathrm{~mm}$, respectively. (b) The magnetic flux density inside the solenoid coil was calculated using commercial software. Axial and radial directions were visualized in a solenoid coil cross-section view. (c) The sinusoidal magnetic flux density was measured using a three-axis sensor. (d) The threedimensional graph describes the measured magnetic flux density along the cross-section inside the solenoid coil when it was set as $1 \mathrm{mT}$. Both color and height ( $z$ axis) indicate the magnetic flux density at each point. 


\section{Real-time PCR}

Total RNA $(1 \mu \mathrm{g})$ extracted from cultured cells at day 7 using TRIzol reagent (Invitrogen, Groningen, The Netherlands) was used as a template for complementary DNA synthesis (Invitrogen, Eugene, OR, USA). Real-time PCR was performed using the SYBR Green PCR Master Mix assay (Applied Biosystems, Warrington, UK) and the ABI StepOnePlus system (Applied Biosystems, Foster City, CA, USA). The amplification reaction consisted of 40 cycles of $95^{\circ} \mathrm{C}$ for $10 \mathrm{~min}$, $95^{\circ} \mathrm{C}$ for $15 \mathrm{~s}$ and $60^{\circ} \mathrm{C}$ for $1 \mathrm{~min}$. The following primers were used: GAPDH sense, 5'-CCAGGTGGTCTCCTCTGACTTC-3'; GAPDH antisense, 5'-GTGGTCGTTGAGGGCAATG-3'; alkaline phosphatase (ALP) sense, 5'-ATGTCATCATGTTCCTGGGAGAT-3'; ALP antisense, $5^{\prime}$-TGGAGCTGACCCTTGAGGAT- $3^{\prime}$; collagen type-I, $\alpha 1$ (COL-I) sense, 5' - CAAGACAGTGATTGAATACAAAACCA-3'; COL-I antisense, 5'- ACGTCGAAGCCGAATTCCT-3'; osteocalcin (OC) sense, 5' - AAGAGACCCAGGCGCTACCT-3'; OC antisense, $5^{\prime}$ - AACTCGTCACAGTCCGGATTG-3'; osterix (OSX) sense, 5' - GG CAGCGTGCAGCAAATT-3'; OSX antisense, 5'- CCTGCTTTGCCC AGAGTTGT-3'; runt-related transcription factor 2 (RUNX2) sense, 5' - AACCCACGAATGCACTATCCA-3'; RUNX2 antisense, 5' - CGGA CATACCGAGGGACATG-3' ${ }^{\prime}$. Gene expression levels of all experimental groups were expressed as fold changes relative to levels of the control group.

\section{ALP activity and staining}

After culture for 10 days, cells were washed three times with phosphate-buffered saline and then lysed in lysis buffer (RIPA buffer, Upstate, Temecula, CA, USA). Cell lysates were incubated with p-nitrophenyl phosphate (Sigma-Aldrich) at $37^{\circ} \mathrm{C}$ for $30 \mathrm{~min}$. The reaction was stopped by adding $2 \mathrm{~N} \mathrm{NaOH}$. Absorbances were measured at $405 \mathrm{~nm}$ using a microplate reader. ALP activity was normalized to the total protein content of each sample. Protein contents were measured using a BCA protein assay kit (Pierce). For ALP staining, cells were fixed with $4 \%$ paraformaldehyde (Wako Pure Chemical, Osaka, Japan). ALP was then stained using a solution containing 0.01\% Naphthol AS-MX phosphate and 0.05\% Fast Red Violet LB Salt (Sigma-Aldrich). Fixed cells were incubated with substrate solution at room temperature for $30 \mathrm{~min}$.

\section{Measurement of mineralization}

Alizarin Red S was used to detect mineralization. Cells were fixed by incubation with $4 \%$ paraformaldehyde for $30 \mathrm{~min}$ at day 20. Samples were then immersed in Alizarin Red S solution at room temperature for $30 \mathrm{~min}$. After washing, stained cells were observed under an optical microscope.

For quantitative calcium measurement, cells were decalcified in $0.6 \mathrm{~N} \mathrm{HCL}$ at day 20 followed by shaking for $24 \mathrm{~h}$ at room temperature. Then, their calcium content was measured using a QuantiChrom Calcium Assay kit (BioAssay Systems, Hayward, CA, USA), as previously reported. ${ }^{26}$ Briefly, $5 \mu \mathrm{l}$ of lysate was mixed with $200 \mu \mathrm{l}$ of working solution from the kit and incubated for $20 \mathrm{~min}$. Absorbances were measured at $612 \mathrm{~nm}$ using a microplate reader.

\section{Immunostaining}

After culture with OM for 4 days, cells were fixed with $4 \%$ paraformaldehyde for $30 \mathrm{~min}$, permeabilized with $0.1 \%$ Triton $\mathrm{X}-100$ and blocked with $0.2 \%$ bovine serum albumin for $15 \mathrm{~min}$. They were then incubated with anti-RUNX2 (Santa Cruz, Heidelberg, Germany) (primary antibody). After washing with phosphate-buffered saline, secondary antibody (Alexa 488 anti-rabbit, diluted 1:200) was added and incubated for $1 \mathrm{~h}$ at room temperature. Samples were mounted with antifade reagent (Invitrogen) and observed using a FluoView 1000 confocal microscope (Olympus, Melville, NY, USA). All images were captured without changing the camera settings.

\section{Statistical analysis}

All experiments were performed in triplicate. Data are presented as the mean \pm s.d. Statistical significance was determined by a one-way analysis of variance $(n=3)$. Differences were considered to be statistically significant at ${ }^{*}$ (or \#) $P<0.05$.

\section{RESULTS}

\section{Electromagnetic field identification}

Before evaluating the effects of the electromagnetic field on ASCs, we characterized the electromagnetic field generated by the solenoid coil (Figure 1a). The magnetic flux density was predicted using three-dimensional finite element analysis before development of the coil. The distribution of the magnetic flux density in the cross-section of the coil was
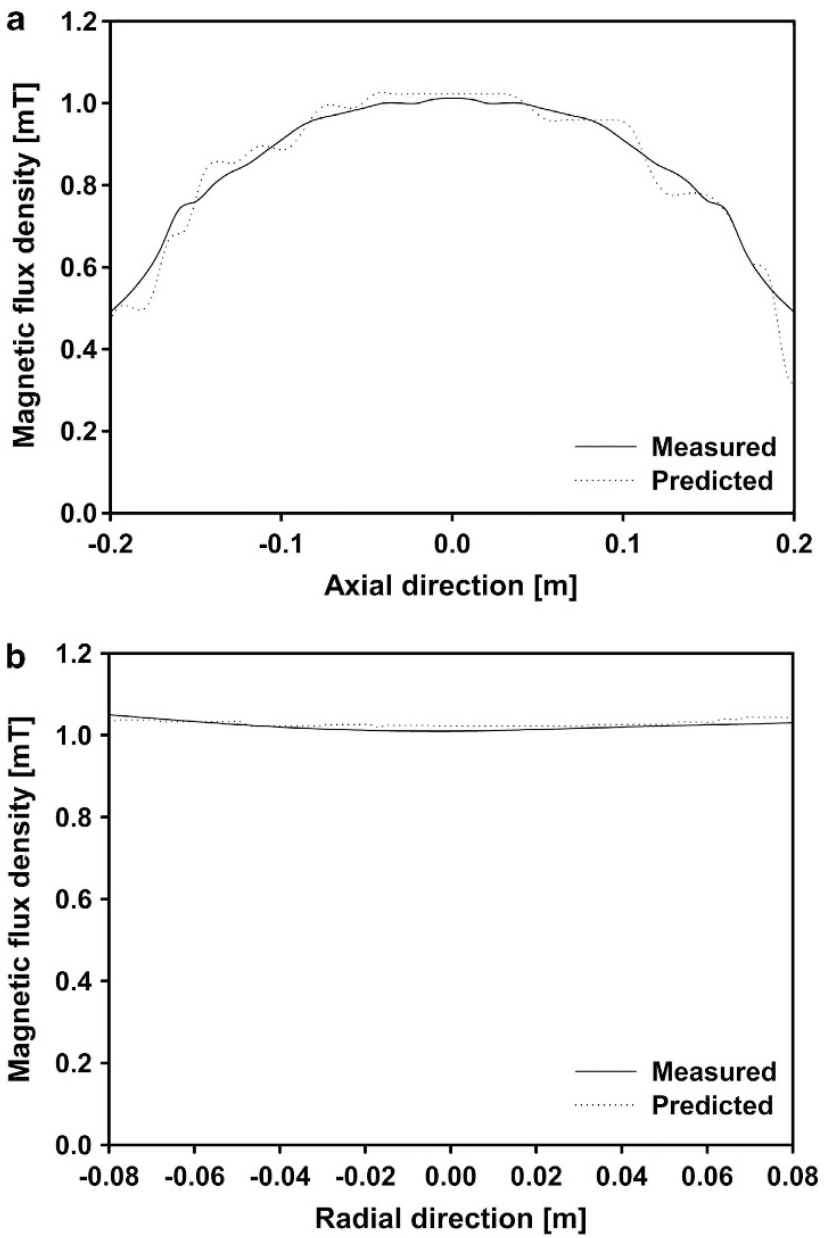

Figure 2 Comparison of the predicted magnetic flux density with the measured data. The predicted magnetic flux density using finite element analysis was similar to the measured values at $1 \mathrm{mT}$. The magnetic flux density was measured and predicted along (a) the axial direction (at point 0 along the radial direction) and (b) the radial direction (at point 0 along the axial direction). 
described in Figure $1 \mathrm{~b}$ when we set the magnetic flux density to $1 \mathrm{mT}$. The magnetic flux density varied throughout the cross-section and was highest at the center along the $y$ axis (axial direction). In contrast, it decreased from both sides (walls) to the center point along the $\mathrm{x}$ axis (radial direction).

The magnetic flux density inside the solenoid coil was measured using a three-axis sensor. As described in Figure 1c, the sinusoidal magnetic flux density generated by the coil was controllable. All measured data along the cross-section of the solenoid coil when it was set to $1 \mathrm{mT}$ are described in a threedimensional graph (Figure 1d). The magnetic flux density at the central area along the axial direction was higher than in the other sections.

This measured magnetic flux density corresponded to predicted values from Figure 1b, as shown in Figure 2. These data were predicted and measured under identical conditions when cells were exposed to the electromagnetic field with an average magnetic flux density of $1 \mathrm{mT}$. We established a nearly uniform area (based on these results) as the experimental section where cells were positioned. This experimental area was at the center along both the axial and radial directions with the dimensions of a commercialized well-plate $(128 \times 86 \mathrm{~mm})$. The average magnetic flux density in this area had a $5 \%$ margin of error.

\section{ASC characterization}

Before inducing osteogenic differentiation, ASCs were characterized using flow cytometry (Figure 3a). ASCs showed negative expression of hematopoietic markers CD34 and CD45. In contrast, ASCs were positive for the cell adhesion molecules CD29 (integrin $\beta_{1}$ ) and CD90 (Thy-1), the metalloproteinase $\mathrm{CD} 13$ (aminopeptidase $\mathrm{N}$ ) and the receptor molecule CD105 (endoglin).

Next, we evaluated the osteogenic potential of ASCs under both the electromagnetic field and OM (Figure 3b). Both the electromagnetic field (electromagnetic field-normal growth medium (NM)) and OM (Control-OM) increased ALP expression and matrix mineralization as compared with the control-NM group. ALP was most strongly activated and mineralized nodules were most heavily formed if ASCs were treated with both the electromagnetic field and $\mathrm{OM}$ simultaneously.
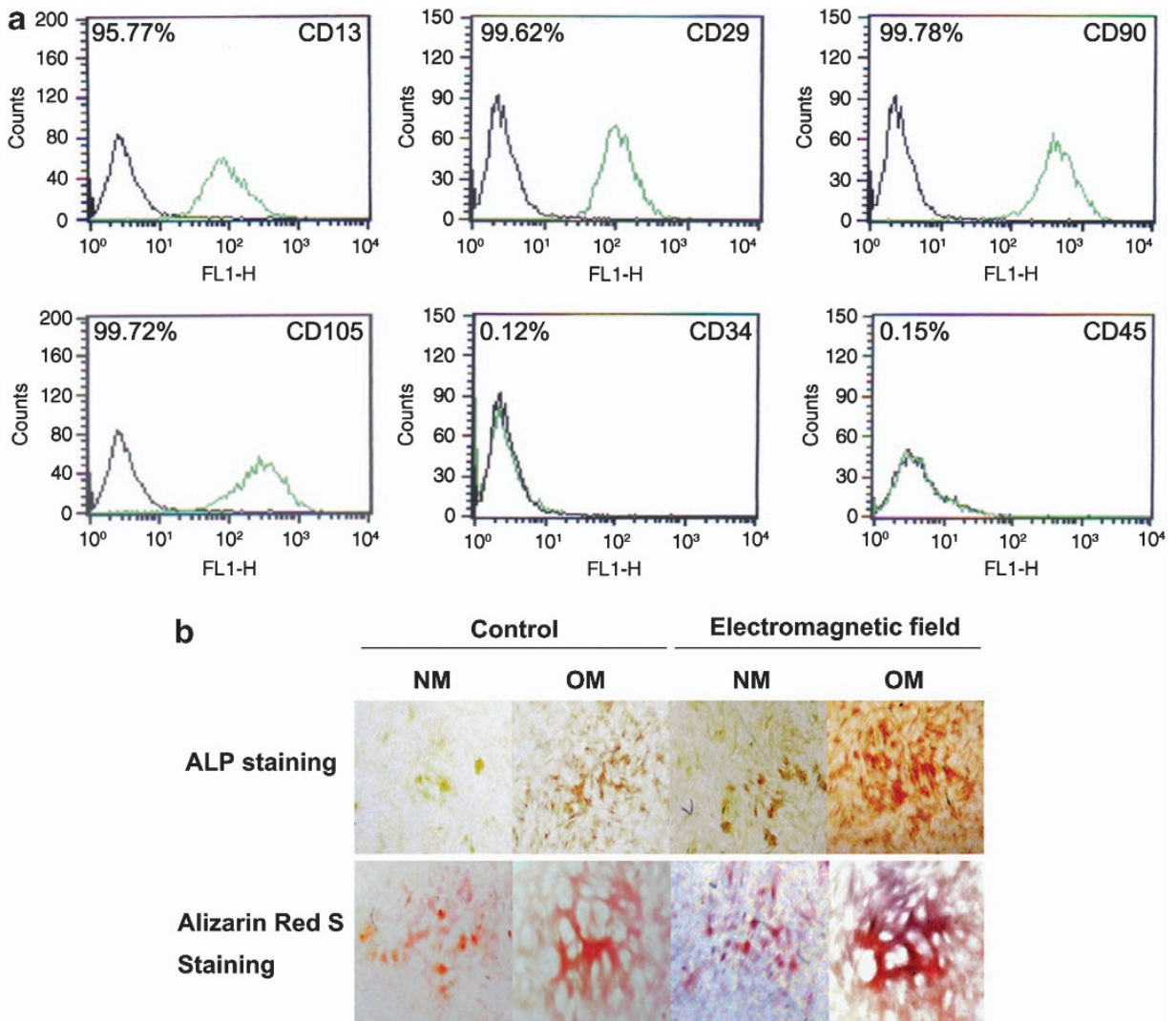

Figure 3 Characterization of adipose-derived stem cells (ASCs). (a) To characterize ASCs, CD (cluster of differentiation) marker proteins were analyzed using fluorescent-conjugated antibodies. ASCs were not hemopoietic (that is, negative for CD34 and CD45), but positive for adhesion (CD29 and CD90), metalloproteinase (CD13) and receptor molecules (CD105). (b) Osteogenic differentiation of ASCs was evaluated by alkaline phosphate (ALP) staining on day 10 and Alizarin Red S staining on day 20. The frequency and magnetic flux density were $30 \mathrm{~Hz}$ and $1 \mathrm{mT}$, respectively. Either osteogenic induction medium (OM) or electromagnetic field induced osteogenic differentiation of ASCs. 


\section{Condition screening}

Relative ALP expression was evaluated using real-time PCR for condition optimization. The magnetic flux density varied from 0.1 to $3 \mathrm{mT}$, and the frequency ranged from 7.5 to $75 \mathrm{~Hz}$. All groups were composed of subgroups (that is, NM; Figure 4a and OM; Figure $4 \mathrm{~b}$ ) to evaluate the synergistic effects of the induction medium and electromagnetic field. ALP expression increased in the frequency range of 30 to $50 \mathrm{~Hz}$ and a magnetic flux density of 0.8 to $2.4 \mathrm{mT}$. The area in red represents the most highly expressed conditions. Frequencies and magnetic flux densities outside of these ranges made no change of ALP expression (blue and violet colors). As shown in Figure 4b, 30 and $45 \mathrm{~Hz}(1 \mathrm{mT})$ increased ALP expression. In contrast, ALP expression was not increased (compared with the control treated with $\mathrm{OM} ; 2.38)$ at $7.5 \mathrm{~Hz}(1$ and $2 \mathrm{mT})$. Overall, we selected three groups to further examine osteogenic differentiation of ASCs; namely, two groups with increased ALP
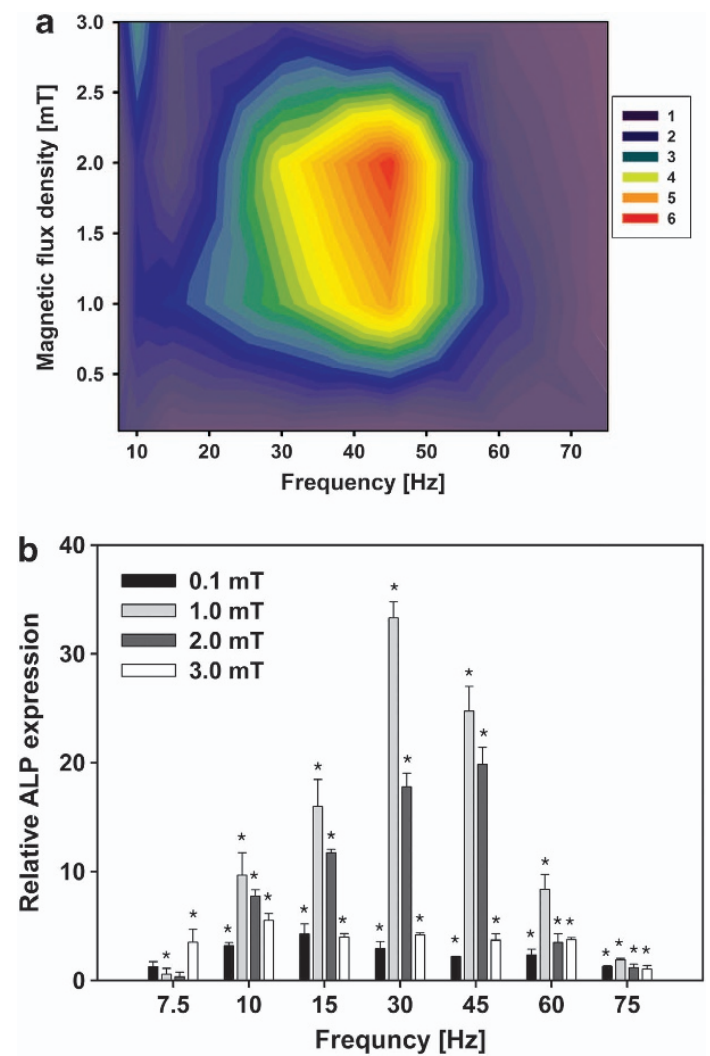

Figure 4 Alkaline phosphate (ALP) expression measured during specific frequency and magnetic flux density conditions. Relative ALP expression was visualized using real-time PCR on day 7 depending on the frequency and magnetic flux density combinations. All levels were normalized to those of the control group exposed to normal growth medium (NM). (a) Adipose-derived stem cells (ASCs) were treated with NM. ALP expression differed according to combinations of the two parameters. Colors indicate the relative ALP expression levels. (b) Relative expression levels of ASCs treated with osteogenic induction medium (OM) are expressed in the bar graph. The control (OM) ALP expression level was 2.38. *Statistically significant differences relative to the control at $P<0.05$. expression $(30 \mathrm{~Hz}(1 \mathrm{mT}), 45 \mathrm{~Hz}(1 \mathrm{mT}))$ and one with decreased ALP expression $(7.5 \mathrm{~Hz}(1 \mathrm{mT}))$.

\section{Confirmation of the selected conditions}

We measured RUNX2 expression using immunostaining to compare positive and negative groups with the control group (Figure 5). RUNX2 expression was increased by OM, as shown in the control group. At $45 \mathrm{~Hz}$, the electromagnetic field strongly induced RUNX2 expression compared with the other groups. At $30 \mathrm{~Hz}$, the electromagnetic field also increased RUNX2 expression compared with the control group. However, RUNX2 expression was reduced compared with the control groups when cells were exposed to a $7.5-\mathrm{Hz}$ electromagnetic field.

We checked RUNX2, COL-I, OSX and OC expression levels using real-time PCR (Figure 6). Expression levels of all genes were increased under the electromagnetic field at 30 and $45 \mathrm{~Hz}$ compared with the control groups. RUNX2 and COL-I levels at $30 \mathrm{~Hz}$ were higher than those at $45 \mathrm{~Hz}$, whereas OSX and OC levels at $30 \mathrm{~Hz}$ were lower than those at $45 \mathrm{~Hz}$. Genes were upregulated by the electromagnetic field at both 30 and $45 \mathrm{~Hz}$ even without OM. Gene expression was not significantly increased at $7.5 \mathrm{~Hz}$ compared with the control with $\mathrm{NM}$.

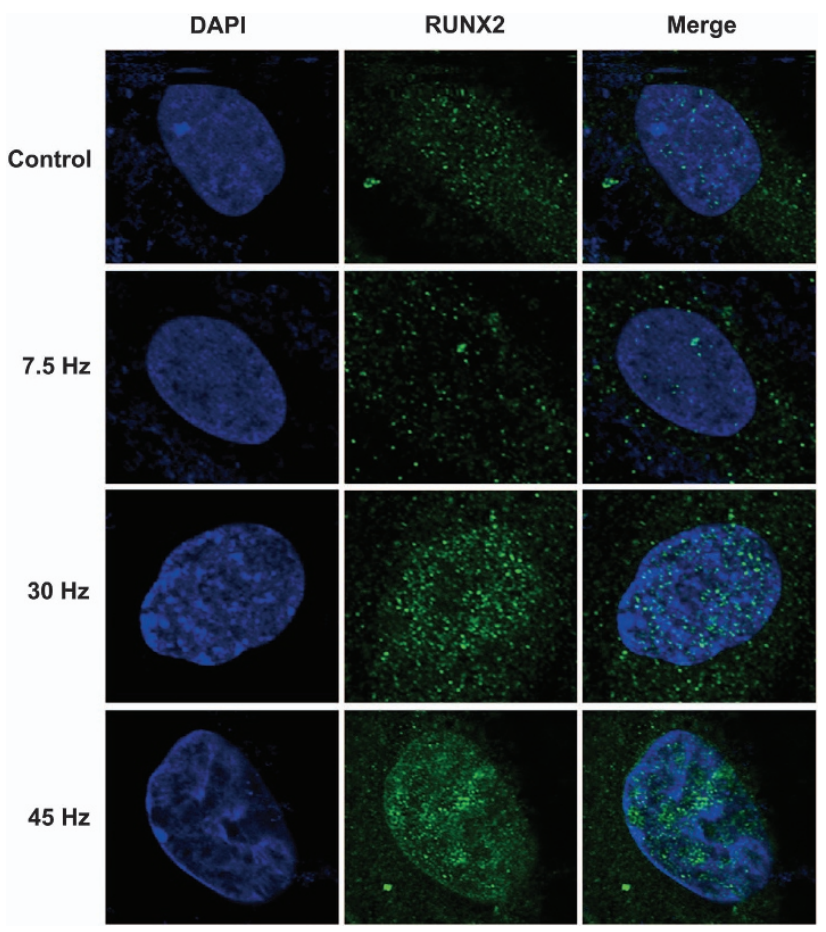

Figure 5 Immunostaining of electromagnetic field induced-RUNX2 (runt-related transcription factor 2) expression. Fluorescence images of RUNX2 expression in the osteogenic induction medium (OM) groups were captured using a confocal microscope on day 4. The 30 and $45 \mathrm{~Hz}$ electromagnetic fields (positive) increased RUNX2 expression, whereas the $7.5 \mathrm{~Hz}$ electromagnetic field (negative) inhibited RUNX2 expression compared with the control group. The magnetic flux density was fixed at $1 \mathrm{mT}$. Original magnification $\times 2000$. 

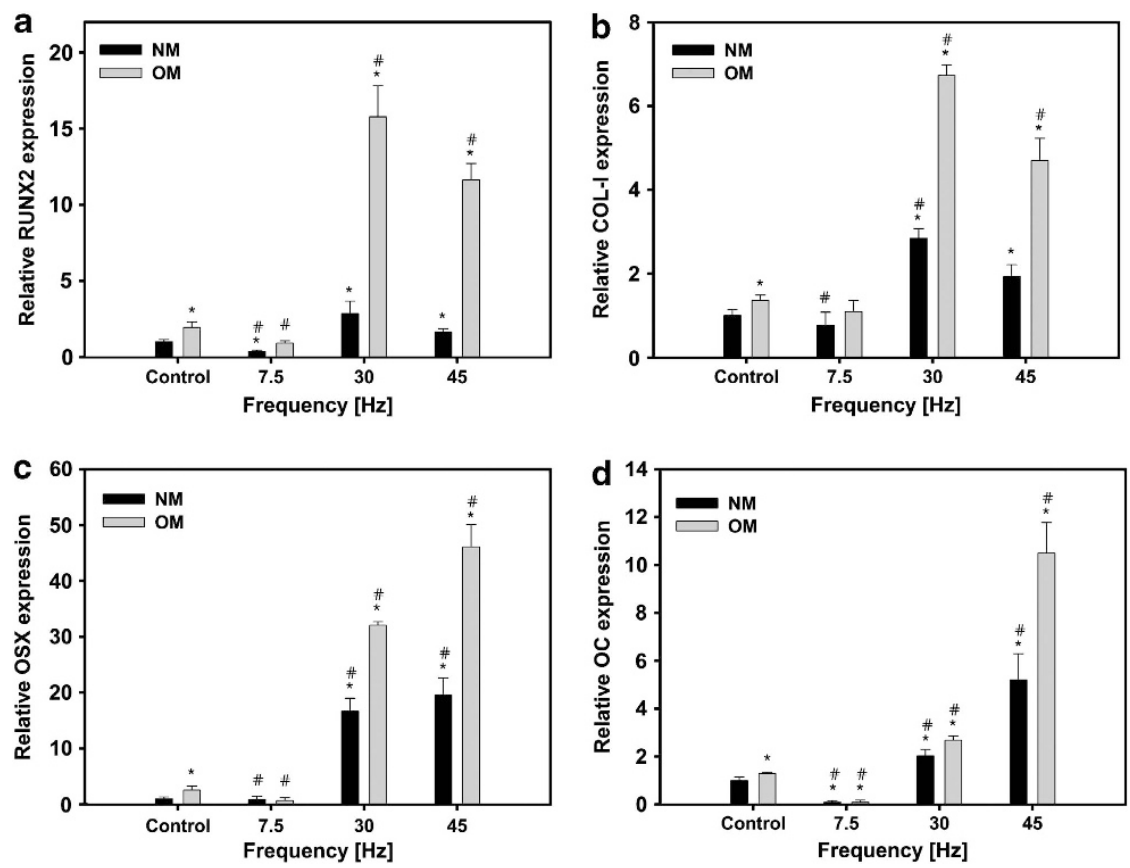

Figure 6 Osteogenic marker expression. Osteogenic marker expression was measured at day 7 using real-time PCR under both normal growth medium (NM) and osteogenic induction medium (OM). (a) Runt-related transcription factor 2 (RUNX2); (b) collagen type-I, $\alpha 1$ (COL-I); (c) osterix (OSX); (d) osteocalcin (OC). ${ }^{*} P<0.05$ (compared with control-NM); ${ }^{\#}<0.05$ (compared with control-OM).

To evaluate the positive and negative effects of the electromagnetic field on the osteogenic differentiation we measured ALP activity (Figure 7a). We found that OM increased ALP activity compared with NM groups, regardless of the electromagnetic field. The ALP activity was higher in the $30 / 45 \mathrm{~Hz}$ groups when ASCs were treated with both $\mathrm{NM}$ and $\mathrm{OM}$ as compared with the control group cultured with identical medium. The activity levels of the $30 / 45 \mathrm{~Hz}$ groups with NM were lower than the control with OM. In contrast, lower ALP activity was detected at $7.5 \mathrm{~Hz}$ as compared with the control group. Although ASCs were treated with OM, the ALP activity at $7.5 \mathrm{~Hz}$ was lower than the control group treated with NM. Matrix production of differentiated osteoblasts was evaluated using a calcium content assay (Figure 7b). Similar to ALP activity, increased amounts of mineralized nodules were detected when ASCs were exposed to $30 / 45 \mathrm{~Hz}$ electromagnetic fields compared with the control group. The calcium levels of the $7.5 \mathrm{~Hz}$ electromagnetic field were lower than the control group. These results suggest that the $30 / 45 \mathrm{~Hz}$ electromagnetic fields stimulated ASC osteogenic differentiation, whereas the $7.5 \mathrm{~Hz}$ electromagnetic field inhibited ASC osteogenic differentiation.

\section{DISCUSSION}

Here, we demonstrated that osteogenic differentiation of ASCs could be regulated by controlling the conditions of an electromagnetic field in vitro. After characterizing the electromagnetic field generated by a solenoid coil, we verified the effects of both positive and negative conditions of the electromagnetic field on osteogenic differentiation of ASCs based on screening results using real-time PCR.

Before screening conditions, it was important to establish the experimental ranges of each parameter based on an osteogenesis literature review. ${ }^{1,2,4-10,12,13,15-17}$ Panagopoulos et al. ${ }^{14}$ proposed the use of a weak electromagnetic field with low frequency based on a biophysical model for the action of oscillating magnetic fields on cells. Additionally, it is known that an electromagnetic field with a frequency below $100 \mathrm{~Hz}$ induces physiological effects as a result of ionic interactions..$^{27,28}$ Thus, based on this information and other previous studies, we established the experimental ranges of the two parameters.

To provide a uniform stimulus to the cells, we first characterized the generated stimulus before evaluating its effects on cells. We estimated the uniform magnetic flux density area of the solenoid coil using finite element analysis to determine dimensions of the solenoid coil with an effective stimulation area. The central area of the coil was reasonably uniform, which is the location of the commercial well plate. Based on these results, an electromagnetic field generator was developed, as described in Figure 1a. When the electromagnetic field was generated inside the solenoid coil, the distributions of the measured data were in good agreement with the estimated values (Figure 2). We established the uniform area with a $5 \%$ margin of error where cells could be exposed to the uniform electromagnetic field.

Before inducing the osteogenic differentiation of ASCs, we confirmed that the ASCs used in this study had typical properties of mesenchymal stem cells, that is, the ASCs 

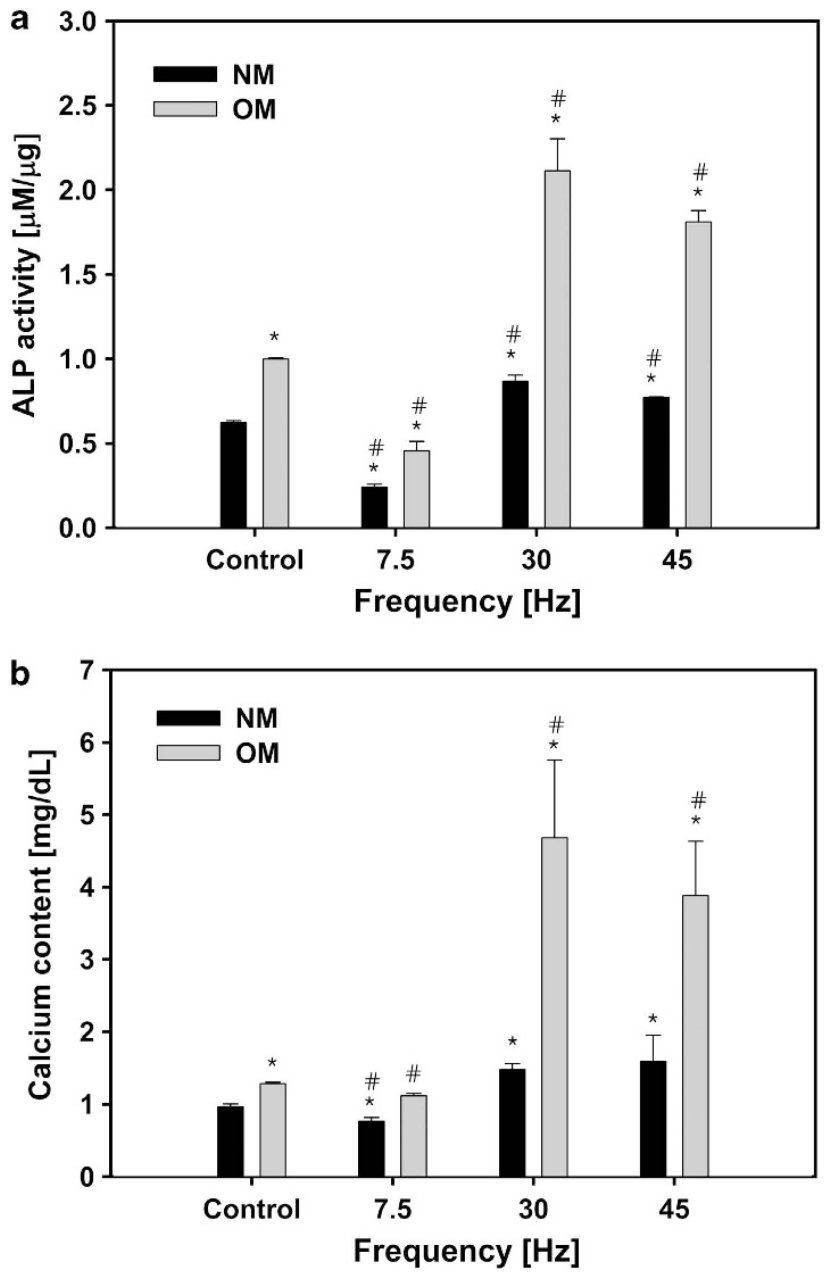

Figure 7 Alkaline phosphate (ALP) activity and matrix mineralization. (a) ALP activity was measured on day 10. (b) Calcium content was measured on day 20 to evaluate matrix mineralization induced by differentiated adipose-derived stem cells (ASCs). The magnetic flux density was fixed at $1 \mathrm{mT}$. ${ }^{*} P<0.05$ (compared with control-NM); ${ }^{\#} P<0.05$ (compared with control-OM). $\mathrm{NM}$, normal growth medium; OM, osteogenic induction medium.

expressed negative hematopoietic markers and positive adhesion/receptor molecules. ${ }^{29-31}$ These ASCs were osteogenically differentiated in the presence of permissive induction medium and electromagnetic field treatment, as illustrated in Figure $3 \mathrm{~b},{ }^{32}$ which indicates that the ASCs used in this study had osteogenic potential that can be accelerated using induction medium and electromagnetic field treatment.

Relative ALP mRNA expression was evaluated using realtime PCR on day 7 to optimize conditions for osteogenic differentiation of ASCs. Two parameters, that is, magnetic flux density and frequency, were controlled independently during the electromagnetic field treatment. When ASCs were stimulated with an electromagnetic field (NM), ALP expression levels were dependent on a combination of magnetic flux density and frequency (Figure 4a). In Figure 4b, ALP was significantly upregulated at a magnetic flux density between 1 and $2 \mathrm{mT}$ and a frequency from 30 to $45 \mathrm{~Hz}$. In contrast, the electromagnetic field did not show positive effects on ALP expression at $7.5 \mathrm{~Hz}$, regardless of the medium type. Taken together, we selected $30 / 45 \mathrm{~Hz}(1 \mathrm{mT})$ and $7.5 \mathrm{~Hz}(1 \mathrm{mT})$ as positive and negative conditions for osteogenic differentiation of ASCs, respectively.

The effect of the selected three conditions on osteogenic differentiation was evaluated using the gene expression of other osteogenic markers. Similar to ALP, RUNX2 is an important early osteogenic marker. ${ }^{33}$ Both the 30 and $45 \mathrm{~Hz}$ groups had increased RUNX2 expression compared with the nonstimulated group, whereas the electromagnetic field at $7.5 \mathrm{~Hz}$ did not increase RUNX2 expression even though cells were treated with OM (Figure 5). Other osteogenic markers were upregulated with the electromagnetic field at both 30 and $45 \mathrm{~Hz}$ compared with the nonstimulated (control) group. However, it was difficult to conclude which frequency had stronger potential to osteogenic differentiation (Figure 6).

ALP activity and calcium content results corroborated the RUNX2 expression data. Although the positive conditions of the electromagnetic field generated synergistic effects with OM on ALP activity and mineralization (Figure 7), the individual effects of $\mathrm{OM}$ on osteogenic differentiation appear stronger than the electromagnetic field. This synergistic effect with the electromagnetic field can be highly induced by other growth factors such as bone morphogenetic protein $2 .{ }^{16}$ Without OM, ALP activities and calcium content in the positive groups were not higher than the control with OM.

The $7.5 \mathrm{~Hz}$ electromagnetic field seemed to inhibit osteogenic differentiation of ASCs. ALP activity at $7.5 \mathrm{~Hz}$ was lower than the control group regardless of the medium type. Calcium content was not increased at this frequency compared with the control groups under the identical medium. Moreover, lower expression levels of other osteogenic markers showed that specific conditions had negative potential to osteogenic differentiation, even though all markers were not inhibited significantly. This inhibitory effect $(7.5 \mathrm{~Hz})$ did not seem to be as strong as the positive effect $(30 / 45 \mathrm{~Hz})$. Based on these data, we conclude that osteogenic differentiation of ASCs can be regulated by controlling the electromagnetic conditions.

Previous papers reported that osteogenic activities can be affected by different frequencies such as 15 or $60 \mathrm{~Hz} .^{12,16,17}$ Similarly, we observed that the electromagnetic field at these frequencies increased ALP expression (Figure $4 \mathrm{~b}$ ). However, our results suggested that frequencies from 30 to $45 \mathrm{~Hz}$ can provide stronger osteogenic effects than either 15 or $60 \mathrm{~Hz}$. This is meaningful because these results were obtained in a single identified system among various conditions to avoid differences caused by experimental and environmental conditions.

Although many questions remain unclear, our study provides a framework for future work to control differentiation lineages of stem cells using biophysical stimulation. Several general mechanisms regarding how electromagnetic fields influence cells have been introduced; analyses suffer from lack of proof on how specific conditions affect osteogenic 
differentiation. ${ }^{14,27,28,34,35}$ We guess that our finding might be related to moving ions with specific frequencies, for example, calcium ions. The ratio of the efflux of $\mathrm{Ca}^{2+}$ through the membrane can be increased at the frequency from 30 to $50 \mathrm{~Hz}^{36}$ Higher efflux of $\mathrm{Ca}^{2}+$ might influence osteoblastic viability and function because the released ions play a structural role of extracellular matrix formation. ${ }^{36-38}$

Treatment using the electromagnetic field could be an auxiliary approach to enhancing cellular activities for tissue regeneration. Stimulation of cells with both electromagnetic field and proper chemical signals will promote the cellular reaction synergistically. Additionally, this inherently noninvasive and noncontact treatment method is easy to be applied to tissue regeneration using three-dimensional scaffolds. ${ }^{39-43}$ Exposure of the electromagnetic field to cells on scaffolds with specific electromagnetic conditions will accelerate tissue formation in vitro.

\section{CONFLICT OF INTEREST}

The authors declare no conflict of interest.

\section{ACKNOWLEDGEMENTS}

This work was supported by the National Research Foundation of Korea (NRF) grant funded by the Korea government (MEST) (No. 2012-0001235) and World Class University (WCU) program through the National Research Foundation of Korea funded by the Ministry of Education, Science and Technology (Project No. R31-2008-00010105-0).

1 Traina GC, Fontanesi G, Costa P, Mammi GI, Pisano F, Giancecchi F et al. Effect of electromagnetic stimulation on patients suffering from non-union. A retrospective study with a control group. Electromagn Biol Med 1991; 10: 101-117.

2 Fredericks DC, Nepola JV, Baker JT, Abbott J, Simon B. Effects of pulsed electromagnetic fields on bone healing in a rabbit tibial osteotomy model. J Orthop Trauma 2000; 14: 93-100.

3 Rubin CT, McLeod KJ, Lanyon LE. Prevention of osteoporosis by pulsed electromagnetic fields. J Bone Joint Surg Am 1989; 71: 411-417.

4 Tabrah F, Hoffmeier M, Gilbert Jr F, Batkin S, Bassett CA. Bone density changes in osteoporosis-prone women exposed to pulsed electromagnetic fields (PEMFs). J Bone Miner Res 1990; 5: 437-442.

5 Lohmann CH, Schwartz Z, Liu Y, Guerkov H, Dean DD, Simon B et al. Pulsed electromagnetic field stimulation of MG63 osteoblast-like cells affects differentiation and local factor production. J Orthop Res 2000; 18 637-646.

6 Tsai MT, Li WJ, Tuan RS, Chang WH. Modulation of osteogenesis in human mesenchymal stem cells by specific pulsed electromagnetic field stimulation. J Orthop Res 2009; 27: 1169-1174.

7 De Mattei M, Caruso A, Traina GC, Pezzetti F, Baroni T, Sollazzo V. Correlation between pulsed electromagnetic fields exposure time and cell proliferation increase in human osteosarcoma cell lines and human normal osteoblast cells in vitro. Bioelectromagnetics 1999; 20: 177-182.

8 Tsai MT, Chang WHS, Chang K, Hou RJ, Wu TW. Pulsed electromagnetic fields affect osteoblast proliferation and differentiation in bone tissue engineering. Bioelectromagnetics 2007; 28: 519-528.

9 Selvarnurugan N, Kwok S, Vasilov A, Jefcoat SC, Partridge NC. Effects of BMP-2 and pulsed electromagnetic field (PEMF) on rat primary osteoblastic cell proliferation and gene expression. J Orthop Res 2007; 25 1213-1220.

10 Fassina L, Visai L, Benazzo F, Benedetti L, Calligaro A, De Angelis MGC et al. Effects of electromagnetic stimulation on calcified matrix production by SAOS-2 cells over a polyurethane porous scaffold. Tissue Eng 2006; 12: 1985-1999.
11 Trock DH, Bollet AJ, Dyer Jr RH, Fielding LP, Miner WK, Markoll R. A double-blind trial of the clinical effects of pulsed electromagnetic fields in osteoarthritis. J Rheumatol 1993; 20: 456-460.

12 Soda A, Ikehara T, Kinouchi Y, Yoshizaki K. Effect of exposure to an extremely low frequency-electromagnetic field on the cellular collagen with respect to signaling pathways in osteoblast-like cells. J Med Invest 2008; 55: 267-278.

13 Midura RJ, Ibiwoye MO, Powell KA, Sakai Y, Doehring T, Grabiner MD et al. Pulsed electromagnetic field treatments enhance the healing of fibular osteotomies. J Orthop Res 2005; 23: 1035-1046.

14 Panagopoulos DJ, Karabarbounis A, Margaritis LH. Mechanism for action of electromagnetic fields on cells. Biochem Biophys Res Co 2002; 298: 95-102.

15 Carson JJ, Prato FS, Drost DJ, Diesbourg LD, Dixon SJ. Time-varying magnetic fields increase cytosolic free $\mathrm{Ca} 2+$ in HL-60 cells. Am J Physiol 1990; 259: C687-C692.

16 Schwartz Z, Simon BJ, Duran MA, Barabino G, Chaudhri R, Boyan BD. Pulsed electromagnetic fields enhance BMP-2 dependent osteoblastic differentiation of human mesenchymal stem cells. J Orthop Res 2008; 26 1250-1255.

17 Sun LY, Hsieh DK, Yu TC, Chiu HT, Lu SF, Luo GH et al. Effect of pulsed electromagnetic field on the proliferation and differentiation potential of human bone marrow mesenchymal stem cells. Bioelectromagnetics 2009 30: 251-260.

18 Gimble JM, Katz AJ, Bunnell BA. Adipose-derived stem cells for regenerative medicine. Circ Res 2007; 100: 1249-1260.

19 Cowan CM, Shi YY, Aalami OO, Chou YF, Mari C, Thomas R et al. Adipose-derived adult stromal cells heal critical-size mouse calvarial defects. Nat Biotechnol 2004; 22: 560-567.

20 Estes BT, Wu AW, Guilak F. Potent induction of chondrocytic differentiation of human adipose-derived adult stem cells by bone morphogenetic protein 6. Arthritis Rheum 2006; 54: 1222-1232.

21 Angle SR, Sena K, Sumner DR, Virdi AS. Osteogenic differentiation of rat bone marrow stromal cells by various intensities of low-intensity pulsed ultrasound. Ultrasonics 2011; 51: 281-288.

22 Halvorsen YD, Franklin D, Bond AL, Hitt DC, Auchter C, Boskey AL et al. Extracellular matrix mineralization and osteoblast gene expression by human adipose tissue-derived stromal cells. Tissue Eng 2001; 7: 729-741.

23 Lee SW, Padmanabhan P, Ray P, Gambhir SS, Doyle T, Contag C et al. Stem cell-mediated accelerated bone healing observed with in vivo molecular and small animal imaging technologies in a model of skeletal injury. J Orthop Res 2009; 27: 295-302.

24 Shoji $T$, Ii M, Mifune $Y$, Matsumoto T, Kawamoto A, Kwon SM et al. Local transplantation of human multipotent adipose-derived stem cells accelerates fracture healing via enhanced osteogenesis and angiogenesis. Lab Invest 2010; 90: 637-649.

25 Hong JM, Kim BJ, Shim JH, Kang KS, Kim KJ, Rhie JW et al. Enhancement of bone regeneration through facile surface functionalization of solid freeform fabrication-based three-dimensional scaffolds using mussel adhesive proteins. Acta Biomater 2012; 8: 2578-2586.

26 Kang KS, Lee SJ, Lee H, Moon W, Cho DW. Effects of combined mechanical stimulation on the proliferation and differentiation of preosteoblasts. Exp Mol Med 2011; 43: 367-373.

27 Gartzke J, Lange K. Cellular target of weak magnetic fields: ionic conduction along actin filaments of microvilli. Am J Physiol-Cell Physio 2002; 283: C1333-C1346.

28 Funk RHW, Monsees TK. Effects of electromagnetic fields on cells: physiological and therapeutical approaches and molecular mechanisms of interaction. Cells Tissues Organs 2006; 182: 59-78.

29 Gronthos S, Franklin DM, Leddy HA, Robey PG, Storms RW, Gimble JM Surface protein characterization of human adipose tissue-derived stromal cells. J Cell Physiol 2001; 189: 54-63.

30 Kolf CM, Cho E, Tuan RS. Mesenchymal stromal cells. Biology of adult mesenchymal stem cells: regulation of niche, self-renewal and differentiation. Arthritis Res Ther 2007; 9: 204.

31 Zuk PA, Zhu M, Ashjian P, De Ugarte DA, Huang JI, Mizuno H et al. Human adipose tissue is a source of multipotent stem cells. Mol Biol Cell 2002 13: 4279-4295.

32 Bunnell BA, Flaat M, Gagliardi C, Patel B, Ripoll C. Adipose-derived stem cells: isolation, expansion and differentiation. Methods 2008; 45 $115-120$ 
33 Lian JB, Javed A, Zaidi SK, Lengner C, Montecino M, van Wijnen AJ et al. Regulatory controls for osteoblast growth and differentiation: role of Runx/ Cbfa/AML factors. Crit Rev Eukar Gene 2004; 14: 1-41.

34 Halgamuge MN, Perssont BRR, Salford LG, Mendis P, Eberhardt J. Comparison between two models for interactions between electric and magnetic fields and proteins in cell membranes. Environ Eng Sci 2009; 26: 1473-1480.

35 Belyaev IY, Alipov ED. Frequency-dependent effects of ELF magnetic field on chromatin conformation in Escherichia coli cells and human lymphocytes. Biochim Biophys Acta 2001; 1526: 269-276.

36 Koch CLMB M, Persson BRR, Salford LG, Eberhardt JL. Interaction between weak low frequency magnetic fields and cell membranes. Bioelectromagnetics 2003; 24: 395-402.

37 Sun XH, McLamore E, Kishore V, Fites K, Slipchenko M, Porterfield DM et al. Mechanical stretch induced calcium efflux from bone matrix stimulates osteoblasts. Bone 2012; 50: 581-591.

38 Boonrungsiman S, Gentleman E, Carzaniga R, Evans ND, McComb DW, Porter AE et al. The role of intracellular calcium phosphate in osteoblastmediated bone apatite formation. Proc Natl Acad Sci USA 2012; 109: 14170-14175.

39 Kim J, Lim D, Kim YH, Koh YH, Lee MH, Han I et al. A comparative study of the physical and mechanical properties of porous hydroxyapatite scaffolds fabricated by solid freeform fabrication and polymer replication method. Int J Precis Eng Man 2011; 12: 695-701.

40 Konrad K, Sevcic K, Foldes K, Piroska E, Molnar E. Therapy with pulsed electromagnetic fields in aseptic loosening of total hip protheses: a prospective study. Clin Rheumatol 1996; 15: 325-328.

$41 \mathrm{Liu}$ FH, Shen YK, Lee JL. Selective laser sintering of a hydroxyapatitesilica scaffold on cultured MG63 osteoblasts in vitro. Int J Precis Eng Man 2012; 13: 439-444.

42 Trock DH. Electromagnetic fields and magnets: Investigational treatment for musculoskeletal disorders. Rheum Dis Clin North Am 2000; 26 : 51-62.

43 Yun JH, Yoo JH, Choi SH, Lee MH, Lee SJ, Song SU et al. Synergistic effect of bone marrow-derived mesenchymal stem cells and platelet-rich plasma on bone regeneration of calvarial defects in rabbits. Tissue Eng Regen Med 2012; 9: 17-23.

This work is licensed under a Creative Commons Attribution-NonCommercial-NoDerivs 3.0 Unported License. To view a copy of this license, visit http:// creativecommons.org/licenses/by-nc-nd/3.0/ 